\title{
Non-canonical gyrotrons
}

\author{
V.E. Zapevalov \\ Institute of Applied Physics RAS, Nizhny Novgorod, Russia, zapev@appl.sci-nnov.ru
}

Scientific research in the 60 s of the last century led to the creation of gyrotron and a number of other gyrodevices, the principle of the its actions are based on the synchronous interaction of the curved electron beam and an electromagnetic wave: the combined effect of relativistic effects and non-homogeneity of high-frequency field on the trajectories of electrons in a magnetic field leads to the intensive cyclotron radiation at the gyrofrequency and its harmonics [1]. The canonical gyrotron version includes adiabatic magnetron-injection electron gun (MIG), forming a tubular helical electron beam (HEB), oversize, weakly irregular cylindrical cavity with a diffraction radiation output, the output window of $\mathrm{rf}$ radiation and electron beam collector. Design of powerful gyrotron as a rule, further includes a built-in quasi-optical mode converter and depressed collector for recovery of the residual energy of the electron beam. The axial symmetry of canonical gyrotron and absence of its small-scale elements are favorable for its industrial development. For small and medium-power gyrotron for technological applications and spectroscopy efficiency increasing and frequency tuning are desirable.

Over the past few years in a number of leading countries created a high level of high-performance powerful gyrotrons from centimeter to submillimeter range [1-3]. Several fundamental scientific fields are formed, the successful development of which is directly caused by the presence of these sources (plasma heating in controlled thermonuclear fusion, development of technology of new materials, spectroscopy (EPR and dynamic nuclear polarization of NMR) and others. Each of the applications dictates own direction of development of gyro-devices, which are analyzed in this paper. For the gyrotron of low power for spectroscopy the customers have high demands for long-term stability and frequency tuning, increase of efficiency. However the complexity of the task is exacerbated by the problem of mode competition and high ohmic losses, especially in gyrotrons operating in the sub millimeter wave range on harmonics of the cyclotron frequency [2-5].

Due to a various reasons during the process of achieving of the maximal energetical characteristics (output power, efficiency, pulse energy), a number of gyro devices, other than the canonical gyrotron version, fizzled out and now were on the sidelines of progress. Such noncanonical devices have partitioned active medium, or (and) the space of interaction; non-cylindrical (ribbon or thin axial) helical electron beam (HEB); oversized strongly non-cylindrical or coaxial, quasi-optical, echelette cavities, etc. However, in the canonical gyrotron are insufficient selective properties at work on the gyro frequency harmonics and very limited possibilities for frequency tuning. The report analyzes the perspectives of non-canonical gyrotron in terms of frequency tuning and improved mode selection working on the gyro frequency harmonics.
Separation of an electron flow for several partial beams give us the possibility to improve mod selection, assisting for operating mode or suppressing parasitic modes. Fig. 1 shows photo of the electron gun for double beam second harmonic gyrotron and experimental dependencies of output power and efficiency on total beam current. Another versions of multi-beams and multi-barrel gyrotrons are discussed also.


Fig. 1. Photo of double beam magnetron injection gum and dependencies of output power and efficiency on total beam current with different current of additional electron beam

Longitudinal sectioning of operation space is an effective way to ensure the selection and efficiency in microwave devices. The gyro-devices partitioning interaction space represented gyro-klystron, double-cavity gyrotron, gyrotrons with coupled cavities (CC), gyromultiplier, etc. In connection with the mode selection problem it may be interesting to use the coupled cavity with mode conversion (CCMC) [5], shown schematically in Fig. 2.

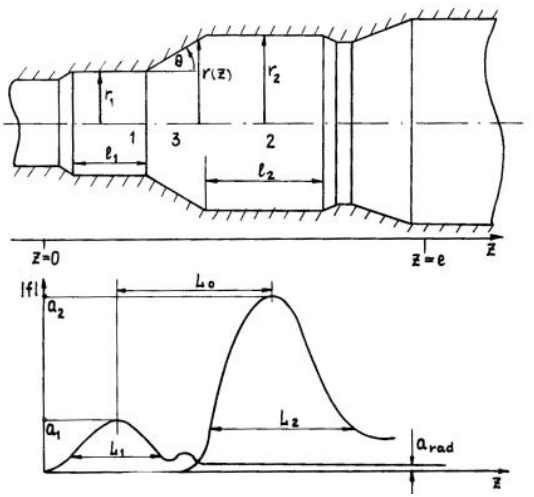

Fig. 2. Scheme and RF field structure of CCMC

The mutual modes conversion in both cavities plays a major role. Resonance Q-enhancement of the normal mode and the amplitude ratio $|\mathrm{a} 1| /|\mathrm{a} 2|$ in CCMC creates basis for high mode selection in these systems and the reduction of radiation on the first mode provides high purity of the output-mode radiation. CCMC used in gyrotrons at the 1-3 harmonics over a broad frequency range, power and pulse duration.

Typical values of efficiency of gyrotrons with CCMC at least do not less than for single cavity gyrotron. These 
data indicate the feasibility of high-performance of CCMC-type gyrotron. Multi CCMC had even higher selectivity properties. As an example, Fig. 3 shows the profile and distribution of the RF field for multi-CCMC gyrotron of average power level with a frequency of $420 \mathrm{GHz}$ at the third harmonic of the gyrofrequency and results of calculation of the output power, efficiency and ohmical load. Disadvantages of CCMC include the need for high-precision manufacturing. Possibility of frequency tuning for these systems is practically absent.
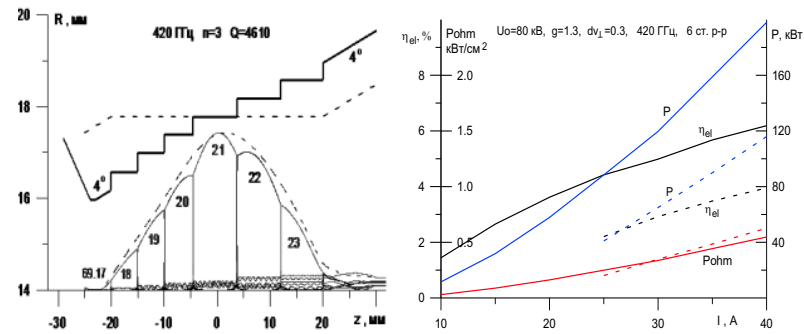

Fig. 3. Profile and RF field distribution of the multi-CCMC average power gyrotron and calculation data of the power, efficiency and ohmical load

Axially symmetric echelette cavity [6] on a some point of view is the development of multi-cavity systems [5] and has similar advantages and disadvantages. It has high selective properties and gyrotron in which such cavity was used in the experiments showed good output characteristics. Eshelette type gyrotron has great potential, but its proper numerical simulation and manufacturing techniques encounter serious problems. The complex structure of the output radiation requires the development of a new version of the QO converter. For some applications may be interested in two-mirror version eshelette gyrotron [6] shown in Fig. 4.

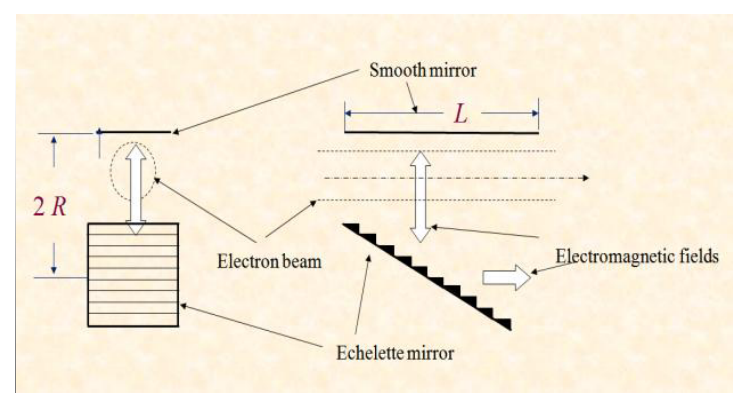

Fig. 4. Two-mirror version eshelette gyrotron

In this gyrotron version there is some possibility of frequency tuning and simplified radiation output system, but using a cylindrical beam inevitably lead to reduction of efficiency. In fact, this scheme is the development of gyrotron with quasi-optical cavity [2, 5], with the additional selection by using eshelette mirror.

Coaxial cavity draw attention of developers of gyrodevices, since its application is promising for achieving of high power levels which favor by high selectivity and absence of voltage depression of electron beam, the possibility of implementing the natural recovery scheme and frequency tuning [3]. Additional features are provided by selection of profiling the inner wall of the cavity and the coaxial insert. The achieved power levels of coaxial gyrotron exceed $2 \mathrm{MW}$ at frequencies up to $170 \mathrm{GHz}$. Using a coaxial gyrotron are complicated due to technological problems associated with mounting, alignment and cooling of coaxial insert.

Great prospects for work on the cyclotron frequency harmonics have a gyrotron in a thin axial beam, often referred to as large-orbit gyrotron (LOG). In experiments with LOG with permanent magnet (field of about $1 \mathrm{~T}$ ) was obtained [7] on these harmonics/ modes / frequency / power / efficiency: $\mathrm{n}=3 / \mathrm{TE}_{311} / 84.88 \mathrm{GHz} / 2.5 \mathrm{~kW} /$ / 6.25\%; $\mathrm{n}=4$ / $\mathrm{TE}_{411} / 112.7 \mathrm{GHz} / 0.47 \mathrm{~kW} / 0.96 \%$. There was also observed oscillation at the 5 th harmonic with frequency $138 \mathrm{GHz}$.

The LOG with pulse magnet [7] $2 \mathrm{~kW}$ power have been achieved at a frequency of around $0.6 \mathrm{THz}(\mathrm{n}=2)$, $2 \mathrm{~kW}$ and $0.3 \mathrm{~kW}$ at a frequency of approximately $1 \mathrm{THz}$ $(\mathrm{n}=3)$ on the 2 nd and 3rd harmonics of the cyclotron frequency respectively.

\section{Summary}

The report analyzes the perspective of non-canonical version of gyrotron in terms of possibility of frequency tuning and improved mode selection for operation on the gyrofrequency harmonics.

Theoretical and experimental results for millimeter and sub-millimeter range gyrotrons with different types of cavities (CCMC, coaxial, quasi-optical, echelette, etc.) are presented and discussed.

The most attractive versions of non-canonical gyrotrons were pointed out.

This work was supported by the Russian Foundation for Basic Research, grant 15-42-02380.

\section{References}

1. G.S. Nusinovich, M.K.A. Thumm, M.I. Petelin. The Gyrotron at 50: Historical Overview. J Infrared Millimeter Terahz Waves (2014) 35:325-381

2. G. Nusinovich. Introduction to the physics of gyrotrons, The Johns Hopkins University Press, Baltimore and London, 2004.

3. M. Thumm. State-of-the-Art of High Power GyroDevices and Free Electron Masers. KIT Scientific Publishing, 2013.

4. V.E. Zapevalov. Evolution of gyrotrons, Radiophysics and Quantum electronics, 2011, v. LIV, №8-9, pp. 559-572.

5. Sh.E. Tsimring. Electron beams and microwave vacuum electronics, John Wiley and Sons, Inc., Hoboken, New Jersey, 2007.

6. E. Jerby, A. Kesar, M. Korol et al. Cyclotron resonance maser array, IEEE Trans. Plasma Sceince, Vol. 27, pp. 445-455, 1999.

7. V.I. Belousov, et al. Research of the gyrotron with the echelette resonator. 9th Int. Workshop Strong Microwaves and Terahertz Waves: Sources and Applications. Nizhny Novgorod Perm - Nizhny Novgorod, Russia July 24 - 30, 2014 p.166-167.

8. V. Bratman, M. Glyavin, T. Idehara, et al. Review of Sub-Terahertz and Terahertz Gyrodevices at IAP RAS and FIR FU. Int. Journal IEEE Trans. on Plasma Science, vol. 37, issue 1, pp. 36-43, (2009) 